Published in final edited form as:

Curr Opin Crit Care. 2014 April ; 20(2): 148-152. doi:10.1097/MCC.0000000000000077.

\title{
Minimally invasive surgery for intracerebral haemorrhage
}

\author{
Benjamin Barnes, Daniel F. Hanley, and Juan R. Carhuapoma \\ Division of Neurosciences Critical Care, Departments of Neurology, Neurosurgery and \\ Anesthesiology and Critical Care Medicine, Johns Hopkins University School of Medicine, \\ Baltimore, Maryland, USA
}

\begin{abstract}
Purpose of review-Spontaneous intracerebral haemorrhage (ICH) imposes a significant health and economic burden on society. Despite this, ICH remains the only stroke subtype without a definitive treatment. Without a clearly identified and effective treatment for spontaneous ICH, clinical practice varies greatly from aggressive surgery to supportive care alone. This review will discuss the current modalities of treatments for ICH including preliminary experience and investigative efforts to advance the care of these patients.
\end{abstract}

Recent findings-Open surgery (craniotomy), prothrombotic agents and other therapeutic interventions have failed to significantly improve the outcome of these stroke victims. Recently, the Surgical Trial in Intracerebral Haemorrhage (STICH) II assessed the surgical management of patients with superficial intraparenchymal haematomas with negative results. MISTIE II and other trials of minimally invasive surgery (MIS) have shown promise for improving patient outcomes and a phase III trial started in late 2013.

Summary-ICH lacks a definitive primary treatment as well as a therapy targeting surrounding perihematomal oedema and associated secondary damage. An ongoing phase III trial using MIS techniques shows promise for providing treatment for these patients.

\section{Keywords}

intracerebral haemorrhage; minimally invasive surgery; MISTIE trial

\section{INTRODUCTION}

\begin{abstract}
Intracerebral haemorrhage (ICH) comprises 10-15\% of all strokes with an incidence of 24.6 per 100000 person-years, with a growing incidence associated with the use of anticoagulation, antiplatelet agents and an ageing population [1-3]. Despite this, ICH remains the last stroke type without a definitive treatment and contributes to significant morbidity and mortality $[4,5]$. Up to half the patients die within 30 days, often despite
\end{abstract}

\footnotetext{
Correspondence to Juan R. Carhuapoma, MD, Division of Neurosciences Critical Care, Departments of Neurology, Neurosurgery and Anesthesiology and Critical Care Medicine, Johns Hopkins University School of Medicine, $600 \mathrm{~N}$ Wolfe Street/Meyer 8-140, Baltimore, MD 21287, USA. Tel: +1 410955 7481; fax: +1 410614 7903; jcarhua1@jhmi.edu.

Conflicts of interest

Benjamin Barnes has no conflicts of interest.

Daniel F. Hanley has no conflict of interest.

Juan R. Carhuapoma has no conflict of interest.
} 
extensive stays in the ICU. Moreover, those who survive have a high degree of long-term disability $[2,6,7]$.

Treatment of ICH ranges from best medical therapy to aggressive management including a variety of surgical techniques $[2,8]$. However, no acute intervention, surgical or medical, has shown definitive benefit on patient outcomes. The lack of strong evidence-based recommendations to guide practice has resulted in significant heterogeneity with clinical practice ranging from aggressive surgery to supportive care or even palliative care without improvement in outcome [2,8-10].

\section{SURGICAL MANAGEMENT OF INTRACEREBRAL HAEMORRHAGE}

Open craniotomy and hematoma aspiration has long been the mainstay of surgical management. However, multiple trials have failed to show improvements in neurologic function following this form of treatment. These failures have many reasons, including the great heterogeneity in patient and $\mathrm{ICH}$-specific factors, treatment and in definitions of care, for example what constitutes early intervention? Attempts to improve outcomes have aimed at modifying surgical timing and patient selection. Early surgery has been favoured in an attempt to limit hematoma expansion, control mass effect and limit early herniation events. However, such attempts have encountered limitations. In 2001, Morgenstern et al. [11] attempted ultra-early craniotomy and hematoma aspiration within $4 \mathrm{~h}$ of symptom onset. The trial was stopped following an unfavourable interim analysis. This analysis showed a redevelopment of hematoma in $40 \%$ of patients treated with surgery within $4 \mathrm{~h}$ compared with the control group who had surgery within $12 \mathrm{~h}$. In the late surgery group, hematoma reaccumulation was observed in $12 \%$ [11].

Subsequent investigations continued to focus on early surgery within 12-24 h from symptom onset. One of the largest randomized clinical trials to compare open craniotomy with nonsurgical treatment was STICH I. This trial that included 1033 patients failed to show a benefit to surgery compared with conservative medical management when surgery was performed within $24 \mathrm{~h}$. Favourable outcomes were observed in $26 \%$ of patients who had surgery and in $24 \%$ of conservatively managed patients as measured by the Glasgow Outcome Score (GOS) [12]. A subsequent subgroup analysis suggested that a subgroup of patients, those with superficial intracerebral hematomas and no intraventricular haemorrhage (IVH), did benefit from surgery [13]. A smaller randomized controlled trial (RCT) that included 108 patients compared early craniotomy $(<8 \mathrm{~h})$ with nonsurgical treatment. Outcome was better in the surgically treated patients, although mortality was similar in the two groups. The main prognostic variables were the initial neurologic status, hematoma volume and location, and in particular patients with a GCS more than 8, ICH volume less than $80 \mathrm{ml}$ or subcortical ICH benefited most from surgery [14].

Although these studies did not show a benefit to surgery for all cases of ICH, they suggested possible benefit in a subset of ICH patients. This was further supported by a meta-analysis in which individual patient data from eight of the 14 RCTs showed improved outcomes with surgical intervention compared with medical management alone if randomization was undertaken within $8 \mathrm{~h}$ of symptom onset, the volume of the hematoma was $20-50 \mathrm{ml}$, the 
GCS was between 9 and 12, or the patient was between the ages of 50 and 69 years [15]. A correction to this article was recently published; although it originally suggested that early surgery was of benefit, the trial used time to randomization and not time to surgery in the analysis, thus making interpretation of the role of timing in surgery difficult [16].

The STICH II trial was based on the subgroup analysis from STICH I and it was designed to test the hypothesis that open craniotomy would benefit select patients. This multicentre, international randomized clinical trial enrolled 601 conscious patients with superficial lobar ICH measuring 10-100 ml, without IVH, and who were admitted with $48 \mathrm{~h}$ of symptom. Despite enrolling the patients most likely to benefit from open craniotomy, early surgery did not improve outcomes, and $59 \%$ of surgical and $62 \%$ of conservative nonsurgical patients had unfavourable outcomes $\left[17^{\mathbf{m}}\right]$.

There are many reasons, including in trial design, why trials of open surgery failed. Potential outcome modifiers that were not fully controlled for in these studies include the timing of surgery, presurgical treatments, rebleeding, late crossovers to craniotomy and incomplete evacuation or effects of IVH. An additional hypothesis to explain the lack of evidence to support benefit of an open craniotomy for ICH invokes surgical trauma to viable brain tissue during the procedure. This trauma to the brain then negates any potential benefit of ICH evacuation. STICH II focused on superficial hematomas, which should limit the degree of surgically induced injury, but this too did not show a significant improvement in patient outcomes. The lack of clear benefit from open surgery has led to significant interest in minimally invasive surgery (MIS) for the evacuation of deep and superficial acute intracranial hematomas.

\section{DEVELOPMENT OF MINIMALLY INVASIVE SURGERY FOR INTRACEREBRAL HAEMORRHAGE}

The development of MIS predates recent trials in open craniotomy for ICH. Nonetheless, the results of trials examining craniotomy have fuelled repeat interest in the minimally invasive technique as an acute intervention. The use of MIS for ICH started in the 1960s. Since then, several modifications to this method to enhance clot evacuation have been explored. Backlund and von Holst [18] used a mechanically assisted endoscopic approach combined with an Archimedes screw and suction. With this technique, they were able to remove large 'densely coagulated' clot [18]. This method was refined by Kandel and Peresedov [19] who were able to achieve near complete removal of clot in 28 of 32 cases, though there were three episodes of hematoma reaccumulation and seven patient deaths in the 2 weeks following surgery. In the late 1980s, mechanically assisted thrombolysis made use of ultrasound techniques and favourable outcomes were described [20].

MIS techniques continued to evolve with the next modification being the addition of thrombolytic infusion through stereotactically placed catheters. For example, Niizuma and Suzuki [21] described 11 patients in which they drained the hematoma and then instilled urokinase in posterior fossa haemorrhages. Of the 11 patients, seven had functional outcomes, and the only patient death was associated with pneumonia [21]. Since then, several investigators have observed that thrombolysis with recombinant tissue-type 
plasminogen activator (rt-PA) is well tolerated and effective in removing clot $[22,23,24$, 25-31]. Pharmacologic thrombolysis has also been combined with sonothrombolysis, and preliminary studies in which recombinant tissue plasminogen activator (tPA) and $24 \mathrm{~h}$ of continuous ultrasound were stereotactically delivered through a burrhole to the clot in 33 patients with ICH, with or without IVH, suggest that the combination of these therapies is more effective in clot lysis and removal than rt-PA alone [22].

These early trials demonstrated the effectiveness of MIS in draining ICH, but the question of clinical benefit remained unanswered. The first RCT to examine MIS was published in 1989 and compared endoscopic evacuation versus medical treatment in 100 patients with spontaneous supratentorial ICHs (subcortical, putaminal and thalamic). Endoscopic ICH evacuation did not benefit patients who were older, had deep hematomas or were stuporous or comatose before surgery. However patients with subcortical bleeds who underwent surgery had reduced mortality, and surgical patients with ICHs less than $50 \mathrm{ml}$ had better functional recovery, but not reduced mortality, than medically treated patients [32]. The results of this study must be interpreted with caution, as the sample size is small and effects were only observed after subgroup analysis, that is the results likely are subject to bias.

\section{THE MINIMALLY INVASIVE SURGERY AND RECOMBINANT TISSUE-TYPE PLASMINOGEN ACTIVATOR IN INTRACEREBRAL HAEMORRHAGE EVACUATION TRIAL}

The recent evolution of MIS has been associated with improved safety and efficacy because of improved understanding of rt-PA dosing safety and ICH patient stability [28,33]. In particular, it is now well documented that the use of rt-PA in MIS is associated with accelerated clot thrombolysis without an increased risk of haemorrhage or other adverse events [24 ${ }^{-25}$ ]. The success of rt-PA in these early studies led to MISTIE II, a phase II multisite trial to examine rt-PA and MIS in spontaneous ICH. The first phase of this trial showed that $1 \mathrm{mg}$ of rt-PA was as well tolerated as $0.3 \mathrm{mg}$ dosed every $8 \mathrm{~h}$. Patients were randomized into two groups, 42 to medical treatment in accordance with published American Heart Association guidelines, and 79 to surgery (69 who received MIS and rt-PA and 10 who had MIS alone). In an initial analysis, clot reduction was greater in the surgical group with an average hematoma volume for surgical patients of 19.6 compared with 40.7 $\mathrm{ml}$ in the medical group. Perihematoma oedema (PHE) was also less in the surgical group with a positive correlation between PHE reduction and percentage of ICH removed. The amount of PHE reduction was similar in MIS alone or MIS and rt-PA patients, that is pharmacological thrombolysis does not appear to have any neurotoxic effects.

\section{THE ROLE OF PERIHEMATOMAL OEDEMA AND PATIENT OUTCOMES}

MISTIE II confirmed that MIS using a catheter-based approach and rt-PA is feasible in properly selected ICH patients and reduced the extent of PHE.

The relationship between PHE and outcomes however is not fully understood. Several studies demonstrate that the volume of PHE is associated with the ICH initial volume, 
volume on a given day and expansion [27,34,35]. Subsequent studies, including MISTIE II, have shown that reduction in ICH volume is associated with reduced PHE [23,25]. However, there are currently no specific therapies targeted towards this oedema $[25,36]$.

The relationship between PHE and long-term tissue injury is more complex, but PHE is associated with poor outcome [37-39], whereas an increase in PHE is associated with a decline in neurologic status [34]. Although MIS is not directed specifically at PHE, its reduction through an approach such as that used in MISTIE II provides an encouraging possibility for improved patient outcome. Ongoing clinical trials, for example MISTIE III and intraventricular thrombolysis with tPA (CLEAR III), are intended to assess the clinical efficacy of these treatments in ICH patients.

There also are ongoing studies to investigate the significance and mechanism of PHE following ICH. MRI studies have been used to try and identify the sequence or 'natural history' and the specific metabolic derangements associated with PHE and soon after hematoma formation suggest the presence of perihematoma inflammation rather than ischemia. This inflammation and/or direct mass effect from the haemorrhage itself may be responsible for reduced cerebral perfusion that has been observed to develop over time [40]. Lysis of red blood cells, thrombin production and other haemostatic events catalysed by platelet activation lead to inflammation adjacent to ICH and have all been implicated in the disruption of the blood-brain barrier that then contributes to ICH. These inflammatory cascades should plausibly play a role in secondary injury following ICH and, as such, represent important therapeutic targets that warrant further investigation [41 $\mathbf{m}$ ].

\section{CONCLUSION}

STICH I, a multicentre RCT, showed that open craniotomy does not improve outcome over best medical management for deep ICH. There may be possibility for benefit in patients with superficial ICH within $1 \mathrm{~cm}$ of the cortical surface. However, the results of STICH II leave unanswered questions about the management of superficial $\mathrm{ICH}$, where in some patients, medical management appears equally effective. The lack of clear clinical benefit of open surgical techniques for ICH has led to the development of minimally invasive surgical techniques including endoscopy and catheter-based drainage with or without the use of pharmacologic or ultrasound-induced thrombolysis. Early evidence suggests that MIS techniques may represent a potential beneficial acute intervention for ICH, but further study is still required.

\section{Acknowledgements}

Daniel F. Hanley was supported by NINDS for MISTIE II, CLEAR III and MISTE III. Juan R. Carhuapoma was supported by MISTE II, ATACH II and CLEAR III.

MISTIE II: NIH/NINDS RO1 NSO 46309, ICH Removal: Safety and Efficacy of MIS and rt-PA MISTIE III: NIH/ NINDS 1U01NS080825, ICH Removal: MIS and rt-PA (MISTIE III) CLEAR III: NIH/NINDS 5U01 NS062851, Clot Lysis: Evaluating Accelerated Resolution of IVH Phase III (CLEAR III) ATACH: NIH/NINDS 1U01NS062091, Antihypertensive Treatment of Acute Cerebral Hemorrhage. 


\section{REFERENCES AND RECOMMENDED READING}

Papers of particular interest, published within the annual period of review, have been highlighted as:

of special interest

- of outstanding interest

1. Van Asch CJ, Luitse MJ, Rinkel GJ, et al. Incidence, case fatality, and functional outcome of intracerebral haemorrhage over time, according to age, sex, and ethnic origin: a systematic review and meta-analysis. Lancet Neurol. 2010; 9:167-176. [PubMed: 20056489]

2. Andrews CM, Jauch EC, Hemphill JC, et al. Emergency neurological life support: intracerebral hemorrhage. Neurocrit Care. 2012; 17(Suppl 1):S37-S46. [PubMed: 22965322]

3. Special report from the National Institute of Neurological Disorders and Stroke. Classification of cerebrovascular diseases III. Stroke [Internet]. Apr 1.1990 21:637-676. [Accessed on 20 November 2013] cited 20 November 2013. http://stroke.ahajournals.org/cgi/doi/10.1161/01.STR.21.4.637.

4. Hanley DF, Awad IA, Vespa PM, et al. Hemorrhagic stroke: introduction. Stroke. 2013; 44(6 Suppl 1):S65-S66. [PubMed: 23709734]

5. Mayer, S., Martin, N., Aronowski, J., et al. [Accessed on 29 October 2013] Final report of the stroke progress review group: topic area working group full reports. 2013. http://www.ninds.nih.gov/ find_people/groups/stroke_prg/2012-stroke-prg-full-report.htm

6. Sacco S, Marini C, Toni D, et al. Incidence and 10-year survival of intracerebral hemorrhage in a population-based registry. Stroke. 2009; 40:394-399. [PubMed: 19038914]

7. Broderick JP, Brott TG, Duldner JE, et al. Volume of intracerebral hemorrhage. A powerful and easy-to-use predictor of 30-day mortality. Stroke. 1993; 24:987-993. [PubMed: 8322400]

8. Broderick J, Connolly S, Feldmann E, et al. Guidelines for the management of spontaneous intracerebral hemorrhage in adults: 2007 update: a guideline from the American Heart Association/ American Stroke Association Stroke Council, High Blood Pressure Research Council, and the Quality of Care and Out. Circulation. 2007; 116:e391-e413. [PubMed: 17938297]

9. Balami JS, Buchan AM. Complications of intracerebral haemorrhage. Lancet Neurol. 2012; 11:101118. [PubMed: 22172625]

10. Hemphill JC, Bonovich DC, Besmertis L, et al. The ICH score: a simple, reliable grading scale for intracerebral hemorrhage editorial comment: a simple, reliable grading scale for intracerebral hemorrhage. Stroke. 2001; 32:891-897. [PubMed: 11283388]

11. Morgenstern LB, Demchuk AM, Kim DH, et al. Rebleeding leads to poor outcome in ultra-early craniotomy for intracerebral hemorrhage. Neurology. 2001; 56:1294-1299. [PubMed: 11376176]

12. Mendelow AD, Gregson BA, Fernandes HM, et al. Early surgery versus initial conservative treatment in patients with spontaneous supratentorial intracerebral haematomas in the International Surgical Trial in Intracerebral Haemorrhage (STICH): a randomised trial. Lancet. 2005; 365:387397. [PubMed: 15680453]

13. Bhattathiri PS, Gregson B, Prasad KSM, Mendelow AD. Intraventricular hemorrhage and hydrocephalus after spontaneous intracerebral hemorrhage: results from the STICH trial. Acta Neurochir Suppl. 2006; 96:65-68. [PubMed: 16671427]

14. Pantazis G, Tsitsopoulos $P$, Mihas $C$, et al. Early surgical treatment vs conservative management for spontaneous supratentorial intracerebral hematomas: a prospective randomized study. Surg Neurol. 2006; 66:492-501. discussion 501-502. [PubMed: 17084196]

15. Gregson BA, Broderick JP, Auer LM, et al. Individual patient data subgroup meta-analysis of surgery for spontaneous supratentorial intracerebral, hemorrhage. Stroke. 2012; 43:1496-1504. [PubMed: 22511006]

16. American Heart Association. Correction. Stroke [Internet]. 2013; 44:e82-e82. [Accessed on 26 December 2013] http://stroke.ahajournals.org/cgi/doi/10.1161/STR.0b013e31829f6281.

17ロ. Mendelow AD, Gregson BA, Rowan EN, et al. Early surgery versus initial conservative treatment in patients with spontaneous supratentorial lobar intracerebral haematomas (STICH II): 
a randomised trial. Lancet. 2013; 382:397-408. [PubMed: 23726393] This is the STICH II trial, a negative study; it showed no benefit for patients undergoing surgery for superficial ICH. This observation has stimulated further interest in MIS as a potential option for the treatment of ICH.

18. Backlund EO, von Holst H. Controlled subtotal evacuation of intracerebral haematomas by stereotactic technique. Surg Neurol. 1978; 9:99-101. [PubMed: 343283]

19. Kandel EI, Peresedov VV. Stereotaxic evacuation of spontaneous intracerebral hematomas. J Neurosurg. 1985; 62:206-213. [PubMed: 3881565]

20. Hondo H, Uno M, Sasaki K, et al. Computed tomography controlled aspiration surgery for hypertensive intracerebral hemorrhage. Experience of more than 400 cases. Stereotact Funct Neurosurg. 1990; 54-55:432-437.

21. Niizuma H, Suzuki J. Computed tomography-guided stereotactic aspiration of posterior fossa hematomas: a supine lateral retromastoid approach. Neurosurgery. 1987; 21:422-427. [PubMed: 3313096]

22. Newell DW, Shah MM, Wilcox R, et al. Minimally invasive evacuation of spontaneous intracerebral hemorrhage using sonothrombolysis. J Neurosurg. 2011; 115:592-601. [PubMed: 21663412]

23. Vespa P, McArthur D, Miller C, et al. Frameless stereotactic aspiration and thrombolysis of deep intracerebral hemorrhage is associated with reduction of hemorrhage volume and neurological improvement. Neurocrit Care. 2005; 2:274-281. [PubMed: 16159075]

24ㅁ. Mould WA, Carhuapoma JR, Muschelli J, et al. Minimally invasive surgery plus recombinant tissue-type plasminogen activator for intracerebral hemorrhage evacuation decreases perihematomal edema. Stroke. 2013; 44:627-634. [PubMed: 23391763] This study is the phase II trial for MIS with rt-TPA for ICH. Phase three of this trial is ongoing. This study found that rtPA with MIS was well tolerated and effective in evacuating clot from patients with MIS.

25. Carhuapoma JR, Barrett RJ, Keyl PM, et al. Stereotactic aspiration-thrombolysis of intracerebral hemorrhage and its impact on perihematoma brain edema. Neurocrit Care. 2008; 8:322-329. [PubMed: 18327659]

26. Naval NS, Nyquist P, Carhuapoma JR. ICH aspiration and thrombolysis. J Neurol Sci. 2007; 261:80-83. [PubMed: 17524425]

27. Barrett RJ, Hussain R, Coplin WM, et al. Frameless stereotactic aspiration and thrombolysis of spontaneous intracerebral hemorrhage. Neurocrit Care. 2005; 3:237-245. [PubMed: 16377836]

28. Naff N, Williams MA, Keyl PM, et al. Low-dose recombinant tissue-type plasminogen activator enhances clot resolution in brain hemorrhage: the intraventricular hemorrhage thrombolysis trial. Stroke. 2011; 42:3009-3016. [PubMed: 21868730]

29. Thiex R, Rohde V, Rohde I, et al. Frame-based and frameless stereotactic hematoma puncture and subsequent fibrinolytic therapy for the treatment of spontaneous intracerebral hemorrhage. $\mathrm{J}$ Neurol. 2004; 251:1443-1450. [PubMed: 15645342]

30. Schaller C, Rohde V, Meyer B, Hassler W. Stereotactic puncture and lysis of spontaneous intracerebral hemorrhage using recombinant tissue-plasminogen activator. Neurosurgery. 1995; 36:328-333. discussion 333-335. [PubMed: 7731513]

31. Rohde V, Rohde I, Thiex R, et al. Fibrinolysis therapy achieved with tissue plasminogen activator and aspiration of the liquefied clot after experimental intracerebral hemorrhage: rapid reduction in hematoma volume but intensification of delayed edema formation. J Neurosurg. 2002; 97:954962. [PubMed: 12405387]

32. Auer LM, Deinsberger W, Niederkorn K, et al. Endoscopic surgery versus medical treatment for spontaneous intracerebral hematoma: a randomized study. J Neurosurg. 1989; 70:530-535. [PubMed: 2926492]

33. Naff NJ, Hanley DF, Keyl PM, et al. Intraventricular thrombolysis speeds blood clot resolution: results of a pilot, prospective, randomized, double-blind, controlled trial. Neurosurgery. 2004; 54:577-584. [PubMed: 15028130]

34. Venkatasubramanian C, Mlynash M, Finley-Caulfield A, et al. Natural history of perihematomal edema after intracerebral hemorrhage measured by serial magnetic resonance imaging. Stroke. 2011; 42:73-80. [PubMed: 21164136] 
35. Carhuapoma JR, Hanley DF, Banerjee M, Beauchamp NJ. Brain edema after human cerebral hemorrhage: a magnetic resonance imaging volumetric analysis. J Neurosurg Anesthesiol. 2003; 15:230-233. [PubMed: 12826970]

36. Poungvarin N, Bhoopat W, Viriyavejakul A, et al. Effects of dexamethasone in primary supratentorial intracerebral hemorrhage. N Engl J Med. 1987; 316:1229-1233. [PubMed: 3574383]

37. Gebel JM, Jauch EC, Brott TG, et al. Relative edema volume is a predictor of outcome in patients with hyperacute spontaneous intracerebral hemorrhage. Stroke. 2002; 33:2636-2641. [PubMed: 12411654]

38. Appelboom G, Bruce SS, Hickman ZL, et al. Volume-dependent effect of perihaematomal oedema on outcome for spontaneous intracerebral haemorrhages. J Neurol Neurosurg Psychiatry. 2013; 84:488-493. [PubMed: 23345281]

39. Arima H, Wang JG, Huang Y, et al. Significance of perihematomal edema in acute intracerebral hemorrhage: the INTERACT trial. Neurology. 2009; 73:1963-1968. [PubMed: 19996072]

40. Carhuapoma JR, Wang P, Beauchamp NJ, et al. Diffusion-perfusion MR evaluation and spectroscopy before and after surgical therapy for intracerebral hemorrhage. Neurocrit Care. 2005; 2:23-27. [PubMed: 16174964]

41․ Ziai WC. Hematology and inflammatory signaling of intracerebral hemorrhage. Stroke. 2013; 44(6 Suppl 1):S74-S78. [PubMed: 23709738] This is a review article of the various mechanisms by which inflammation and perihematomal oedema may play a role in secondary damage following ICH. These inflammatory cascades represent potential points for intervention to prevent secondary damage following ICH. 


\section{KEY POINTS}

- There is currently no surgical treatment for acute intracerebral haemorrhage (ICH) that has been shown to improve patient outcome.

- Open surgical procedures to treat acute ICH have failed to show clinical benefit in large multicentre RCTs despite earlier timing, careful patient selection and limiting surgery to superficial ICH, and hence, for most patients, medical therapy appears as effective as open craniotomy.

- Minimally invasive surgery using a variety of methods, including endoscopic evacuation, catheter based approaches, ultrasound and thrombolytics alone or in combination, is feasible and clinical studies show that MIS is effective in ICH evacuation.

- $\quad$ Ongoing phase III trials will evaluate whether MIS is associated with clinical benefit. 